\title{
Sleep Spindles and Intelligence: Evidence for a Sexual Dimorphism
}

\author{
Péter P. Ujma, ${ }^{1}$ Boris Nikolai Konrad, ${ }^{2}$ Lisa Genzel, ${ }^{3}$ Annabell Bleifuss, ${ }^{4}$ Péter Simor, ${ }^{5,7}$ Adrián Pótári, ${ }^{1}$ \\ János Körmendi, ${ }^{1}$ Ferenc Gombos, ${ }^{6}$ Axel Steiger, ${ }^{4}{ }^{\odot}$ Róbert Bódizs, ${ }^{1,6 *}$ and ${ }^{\oplus}$ Martin Dresler ${ }^{2,4 *}$ \\ ${ }^{1}$ Institute of Behavioral Science, Semmelweis University, H-1089 Budapest, Hungary, ${ }^{2}$ Donders Institute for Brain, Cognition and Behaviour, Radboud \\ University Medical Centre, 6525 EN Nijmegen, The Netherlands, ${ }^{3}$ Centre for Cognitive and Neural Systems, University of Edinburgh, EH8 9JZ Edinburgh, \\ United Kingdom, ${ }^{4}$ Max Planck Institute of Psychiatry, 80804 Munich, Germany, ${ }^{5}$ Department of Cognitive Sciences, Budapest University of Technology and \\ Economics, 1111 Budapest, Hungary, ${ }^{6}$ Department of General Psychology, Pázmány Péter Catholic University, H-1088 Budapest, Hungary, and 7Nyírö \\ Gyula Hospital, National Institute of Psychiatry and Addictions, H-1135 Budapest, Hungary
}

Sleep spindles are thalamocortical oscillations in nonrapid eye movement sleep, which play an important role in sleep-related neuroplasticity and offline information processing. Sleep spindle features are stable within and vary between individuals, with, for example, females having a higher number of spindles and higher spindle density than males. Sleep spindles have been associated with learning potential and intelligence; however, the details of this relationship have not been fully clarified yet. In a sample of 160 adult human subjects with a broad IQ range, we investigated the relationship between sleep spindle parameters and intelligence. In females, we found a positive age-corrected association between intelligence and fast sleep spindle amplitude in central and frontal derivations and a positive association between intelligence and slow sleep spindle duration in all except one derivation. In males, a negative association between intelligence and fast spindle density in posterior regions was found. Effects were continuous over the entire IQ range. Our results demonstrate that, although there is an association between sleep spindle parameters and intellectual performance, these effects are more modest than previously reported and mainly present in females. This supports the view that intelligence does not rely on a single neural framework, and stronger neural connectivity manifesting in increased thalamocortical oscillations in sleep is one particular mechanism typical for females but not males.

Key words: intelligence; memory; sex; sleep; sleep spindles

\section{Introduction}

Sleep spindles are typical features of nonrapid eye movement (NREM) sleep, arising as an interaction of thalamocortical, corticothalamic, and reticular neurons due to the absence of cholinergic activation during NREM sleep (Steriade and Deschenes, 1984; Amzica and Steriade, 2000; Steriade, 2000; Fogel and Smith, 2011). These oscillations provide excellent conditions for long-term synaptic changes (Buzsáki, 1989; Rosanova and Ulrich, 2005; Fogel and Smith, 2011), and the interplay of spindles and hippocampal ripples plays an important role in neuroplasticity (Clemens et al., 2007; Genzel et al., 2014). Specifically, spindles deafferent the cortex from the hippocampus, enabling local processing of increased firing rates in the cortex in response to hippocampal firing during ripples (Peyrache et al., 2009; Wierzynski et al., 2009; Genzel et al., 2014). Spindle activity indeed

Received April 29, 2014; revised Oct. 18, 2014; accepted Oct. 23, 2014.

Author contributions:P.P.U., R.B., and M.D. designed research;P.P.U., B.N.K., L.G., A.B., P.S., and M.D. performed research; J.K. and F.G. contributed unpublished reagents/analytic tools; P.P.U., A.P., R.B., and M.D. analyzed data; P.P.U., B.N.K., L.G., P.S., A.P., A.S., R.B., and M.D. wrote the paper.

We thank the members of Mensa HungarlQa and Mensa in Deutschland for their assistance in this study.

The authors declare no competing financial interests.

*R.B. and M.D. contributed equally to this work.

Correspondence should be addressed to Dr. Martin Dresler, Max Planck Institute of Psychiatry, Kraepelinstrasse

2-10, 80804 Munich, Germany. E-mail: dresler@mpipsykl.mpg.de.

DOI:10.1523/JNEUROSCI.1857-14.2014

Copyright $\odot 2014$ the authors $\quad 0270-6474 / 14 / 3416358-11 \$ 15.00 / 0$ correlates with memory consolidation in both declarative (Gais et al., 2002; Clemens et al., 2005; Genzel et al., 2009) and procedural (Fogel and Smith, 2006; Fogel et al., 2007; Morin et al., 2008) tasks.

Sleep spindles also reflect trait variables of cognition. Spindle parameters show strong intraindividual stability and are important components of the individual sleep EEG fingerprint (De Gennaro et al., 2005). Individual profiles in spindling reflect the microstructural properties of white matter tracts as measured by diffusion weighted MRI, with high levels of spindling being related to high axial diffusivity in white matter structures (Piantoni et al., 2013). Sleep spindle density correlates with measures of verbal memory (Lafortune et al., 2014), visuospatial memory (Bódizs et al., 2008), selective attention (Forest et al., 2007; Limoges et al., 2013), and fluid intelligence (Bódizs et al., 2005). Intelligence also correlates with the absolute number of spindles (Fogel et al., 2007), and sleep spindle duration and amplitude (Schabus et al., 2006; Fogel et al., 2007), suggesting that the efficiency of thalamocortical tracts reflected by prominent spindle activity is essential for overall cognitive ability.

However, previous studies were performed on relatively small and homogeneous samples (mainly university students of average to moderately high intelligence), rendering interpretation of correlation strength difficult. It is also unclear whether correlates of intelligence are similar throughout the entire IQ range (Fogel 
and Smith, 2011) and whether intelligence continues to correlate with spindle parameters within the extreme range, as correlates of intelligence often decrease with the distance from the average, a phenomenon known as the law of diminishing returns (Spearman, 1927; Tucker-Drob, 2009). Moreover, increased spindle activity has been reported not only in high IQ subjects, but also in children with mental retardation (Gibbs and Gibbs, 1962; Bixler and Rhodes, 1968); and, based on these findings, a U-shaped association between intelligence an sleep spindle activity has been proposed (Fogel et al., 2007; Fogel and Smith, 2011).

Sex differences were reported for the number of sleep spindles, spindle density, and EEG $\sigma$ power (Gaillard and Blois, 1981; Carrier et al., 2001; Huupponen et al., 2002; Genzel et al., 2012) as well as functional correlates of spindles, namely, learning-related increases in spindle activity, which was only present in males and females in their mid-luteal menstrual phase (Genzel et al., 2012). Sex differences were also repeatedly shown for brain structures and neural correlates of cognitive performance (Cahill, 2006; Jazin and Cahill, 2010). Females generally show stronger values in various connectivity measures (Gong et al., 2009; Tomasi and Volkow, 2012; Ingalhalikar et al., 2014; Satterthwaite et al., 2014) and stronger association between white matter and intellectual performance than males (Gur et al., 1999; Haier et al., 2005). We therefore hypothesized that the sleep spindle-IQ relationship is characterized by sexually dimorphic aspects, with females showing stronger correlations between intelligence and spindle activity than males.

\section{Materials and Methods}

We investigated the relationship between sleep spindle parameters and performance in tests of fluid reasoning, controlling for the effects of age and sex in a large sample. A total of 160 subjects ( 72 females, 88 males) participated in this study, in a cooperation between the Max Planck Institute of Psychiatry (Munich, Germany) and the Psychophysiology and Chronobiology Research Group of Semmelweis University (Budapest, Hungary). Mean age of subjects was 29.7 years (SD 10.7 years, range 17-69 years). The sleep spindle database was created using previously existing polysomnography recordings with available IQ scores, which has never been used in publications addressing the relationship between sleep spindles and intelligence, either in its entirety or in part. We further added new recordings specifically to increase the IQ variability within the sample, as our aim was to include subjects from a broad intelligence range. To avoid a masking of potential correlations between spindle parameters and intelligence through the inclusion of subjects with potentially pathological processes, we aimed to widen the intelligence distribution of our sample toward the upper extreme of the IQ range. We therefore recruited a considerable number of subjects among the members of the high-IQ society Mensa. The overall distribution of IQ scores is illustrated by Figure 1.

The research protocols were approved by the Ethical Committee of the Semmelweis University (Budapest, Hungary) or the Medical Faculty of the Ludwig Maximilians University (Munich, Germany) in accordance with the Declaration of Helsinki. All subjects signed informed consent for the participation in the studies. According to semistructured interview with experienced psychiatrists or psychologists, all subjects were healthy, had no history of neurologic or psychiatric disease, and were free of any current drug effects, excluding contraceptives. Consumption of small habitual doses of caffeine (maximum 2 cups of coffee before noon), but no alcohol, was allowed. Six male and 2 female subjects were light to moderate smokers (self-reported), and the rest of the subjects were nonsmokers.

Based on their availability, all subjects completed one or two standardized nonverbal intelligence tests. The tests used in the study were the Culture Fair Test (CFT) (Weiss and Weiss, 2006) and Raven Advanced Progressive Matrices (Raven APM, (Raven et al., 2004). Both the CFT and Raven APM are nonverbal intelligence tests where subjects are re- quired to complete abstract patterns by finding their organizing rules. Performance in these tests was shown to correlate strongly and to be a particularly good measurement of the general factor of intelligence (Cattell, 1973; Duncan et al., 2000; Prokosch et al., 2005). A total of 113 subjects completed the CFT and 89 subjects completed the Raven APM test; 42 subjects completed both tests.

Sleep spindle parameters were expected to change as a factor of age, and IQ scores derived from intelligence tests are age-corrected, whereas raw scores of different intelligence tests are on different scales. Therefore, a composite raw intelligence test score was calculated, expressed as a Raven equivalent score. Raven equivalent scores for Raven APM tests were equal to the actual raw test score. For CFT raw scores, Raven equivalent scores were equal to the Raven APM score corresponding to the IQ percentile derived from CFT performance and the age of the subject, in other words, the Raven APM score that would have yielded the same population percentile score as the actually completed CFT test. If both Raven APM and CFT scores were available for a subject, the two Raven equivalent scores were averaged. Raven APM was chosen as a basis of standardization because of the availability of detailed norms. For this study, norms from the 1993 Des Moines (Iowa) standardization (Raven et al., 2004) of APM were used. Mean Raven equivalent score was 26.8 (SD 6.2, range 10.5-36). There was no difference in age $(F=1.16, p>$ $0.9)$ or Raven equivalent scores $(F=1.36, p>0.1)$ between males (mean age 29.5 years, SD 10.4 years; mean Raven 27.5, SD 5.7) and females (mean age 29.3 years, SD 11.2 years; mean Raven 26.0, SD 6.7).

Sleep was recorded for two consecutive nights by standard polysomnography, including EEG according to the 10-20 system (Jaspers, 1958) (common recording sites across the studies and laboratories were as follows: Fp1, Fp2, F3, F4, Fz, F7, F8, C3, C4, Cz, P3, P4, T3, T4, T5, T6, $\mathrm{O} 1$, and $\mathrm{O} 2$ ), electro-oculography, bipolar submental electromyography, as well as electrocardiography. EEG electrodes were rereferenced to the mathematically linked mastoids. Impedances for the EEG electrodes were kept $<8 \mathrm{k} \Omega$. Signals were collected, prefiltered, amplified, and digitized at different sampling rates using different recording apparatus in the different subsamples (Table 1).

Sleep EEG recordings for the second nights spent in the laboratory were manually scored on a $20 \mathrm{~s}$ basis by applying standard criteria (Iber, 2007). Epochs with artifacts were removed on a $4 \mathrm{~s}$ basis by visual inspection of all recorded channels (including polygraphy). The individual adjustment method (IAM) of sleep spindle analysis was applied for N2 and N3 sleep (Bódizs et al., 2009). The IAM procedure considers the individual spectral peaks as starting points for the sleep spindle analysis. In short, the second-order derivatives of 9-16 Hz amplitude spectra (4 s, Hanning tapered Fast Fourier Transform) of NREM sleep EEGs were averaged over EEG derivations and frequencies corresponding to the zero crossing points encompassing those two negative peaks with the largest absolute amplitudes were defined as frequency criteria for slow and fast sleep spindles. In cases of uncertainty (lack of zero crossing points indicating slow spindles or partial overlap between slow and fast sleep spindles in some cases), frequencies with predominance of power in averaged frontal (Fp1, Fp2, F3, F4, Fz, F7, F8) over averaged centroparietal $(\mathrm{C} 3, \mathrm{C} 4, \mathrm{Cz}, \mathrm{P} 3, \mathrm{P} 4)$ amplitude spectra were considered as slow spindle frequencies $(N=18)$. There was no case of uncertainty related to the frequency boundaries of fast spindles. Resulting slow and fast spindle boundaries were used as frequency limits for slow and fast spindle bandpass filtering (FFT-based, Gaussian filter, $16 \mathrm{~s}$ windows) of the EEGs.

Thresholding of the envelopes of the bandpass filtered EEGs were performed by using individual- and derivation-specific amplitude criteria: means of the amplitude spectral values $(\mu \mathrm{V})$ at the frequencies corresponding to the lower and upper limits of sleep spindling were considered after multiplying by the number of bins making up the band. The envelope of the bandpass filtered signal had to exceed the threshold for at least $0.5 \mathrm{~s}$ to be considered a sleep spindle. Slow and fast sleep spindles defined in this way were counted and characterized by the following sleep spindle parameters:

1. Slow and fast sleep spindle density (spindles/minutes of N2 or N3 sleep, spindles $\min ^{-1}$ ); 
Table 1. Details of the recording procedures in different subsamples

\begin{tabular}{|c|c|c|c|c|c|}
\hline & $N$ & EEG recording sites (10 -20 system) & Polygraphic channels & Electrodes used & $\begin{array}{l}\text { Effective sampling rate/ } \\
\text { sampling rate }(\mathrm{Hz})\end{array}$ \\
\hline Budapest-I & 31 & $\begin{array}{l}\mathrm{Fp} 1, \mathrm{Fp} 2, \mathrm{~F} 3, \mathrm{~F} 4, \mathrm{Fz}, \mathrm{F} 7, \mathrm{~F} 8, \mathrm{C} 3, \mathrm{C} 4, \mathrm{Cz}, \mathrm{P} 3, \mathrm{P} 4 \text {, } \\
\mathrm{T} 3, \mathrm{~T} 4, \mathrm{~T} 5, \mathrm{~T} 6,01,02\end{array}$ & $\begin{array}{l}\text { Left and right EOG, bipolar submental EMG, } \\
\text { ECG, thoracic and abdominal respiration }\end{array}$ & $\begin{array}{l}\text { Au coated } \mathrm{Ag} / \mathrm{AgCl} \text { fixed with EC2 Grass } \\
\text { electrode cream }\end{array}$ & $249 / 249$ \\
\hline Budapest-II & 16 & $\begin{array}{l}\text { Fp1, Fp2, F3, F4, Fz, F7, F8, C3, C4, Cz, P3, P4, } \\
\text { Pz, T3, T4, T5, T6, 01, } 02\end{array}$ & Bipolar EOG, bipolar submental EMG, ECG & $\begin{array}{l}\text { Au coated } \mathrm{Ag} / \mathrm{AgCl} \text { fixed with EC2 Grass } \\
\text { electrode cream }\end{array}$ & $4096 / 1024$ \\
\hline Munich-I & 93 & $\begin{array}{c}\text { Fp1, Fp2, Fpz, AF1, AF2, F3, F4, Fz, F7, F8, C3, } \\
\text { C4, Cz, P3, P4, Pz, T3, T4, T5, T6, 01, } 02\end{array}$ & Bipolar EOG, bipolar submental EMG, ECG & $\begin{array}{l}\mathrm{Ag} / \mathrm{Ag}-\mathrm{Cl} \text {, with EC2 Grass electrode cream for } \\
\text { EEG and Nihon Kohden ELEFIX for EMG }\end{array}$ & $250 / 250$ \\
\hline Munich-II & 20 & Fp1, Fp2, F3, F4, C3, C4, P3, P4, 01, 02 & Bipolar EOG, bipolar submental EMG, ECG & $\begin{array}{l}\mathrm{Ag} / \mathrm{Ag}-\mathrm{Cl} \text {, with EC2 Grass electrode cream for } \\
\text { EEG and Nihon Kohden ELEFIX for EMG }\end{array}$ & $250 / 250$ \\
\hline
\end{tabular}

2. Slow and fast sleep spindle durations (mean duration of sleep spindles detected, seconds);

3. Slow and fast sleep spindle amplitudes (mean maxima of the intraspindle envelopes of bandpass filtered EEGs, $\mu \mathrm{V}$ );

4. The peak frequencies and upper/lower frequency limits of slow and fast spindles for each subject.

To correct for the different analog EEG filter characteristics of our machines, we connected an analog waveform generator to the $\mathrm{C} 3$ and $\mathrm{C} 4$ electrode inputs (with original recording reference, rereferenced for A1-A2 common references for further analysis) of all EEG devices and applied 40 and $355 \mu \mathrm{V}$ amplitude sinusoid signals of various amplitudes $(0.05 \mathrm{~Hz}$, every $0.1 \mathrm{~Hz}$ between $0.1-2 \mathrm{~Hz}$, every $1 \mathrm{~Hz}$ between $2-20 \mathrm{~Hz}$, every $10 \mathrm{~Hz}$ between $10 \mathrm{~Hz}-100 \mathrm{~Hz}$ ).

We determined the amplitude reduction rate of each recording system by calculating the proportion between digital (measured) and analog (generated) amplitudes of sinusoid signals at typical sleep spindle frequencies $(10,11,12,13,14$, and $15 \mathrm{~Hz}$ ) for both inducing ( 40 and $355 \mu \mathrm{V}$ amplitude) signals. Machine-specific amplitude reduction rates were given as the mean amplitude rate between digital and analog values at the two amplitudes and six measured frequencies (for the reduction rates, see Table 1). Sleep spindle amplitudes were corrected by dividing their calculated values by the amplitude reduction rate of the recording system.

Given the individual- and derivation-specific adjustment inherent to the procedure, sleep spindle densities and durations are amplitudeinsensitive measures (for an empirical demonstration, see Bódizs et al., 2005). Thus, there is no need for the compensation of the different recording systems in these values. Group comparisons (male vs female) were performed by independent samples $t$ tests. Partial Pearson correlation coefficients were calculated to test the relationship between sleep spindle parameters and Raven equivalent scores, controlling for the effects of age. This was deemed necessary because of the potential effects of age on both sleep spindle parameters (De Gennaro and Ferrara, 2003; Fogel and Smith, 2011) and intelligence test performance (Tucker-Drob, 2009). To control for multiple comparisons across electrodes, we performed the Benjamini-Hochberg procedure (Benjamini and Hochberg, 1995) controlling for the false discovery rate for each sleep spindle parameter. This correction procedure was selected because sleep spindle parameters at different electrodes are expected to correlate positively, rendering a Bonferroni correction overly conservative. The BenjaminiHochberg procedure, on the other hand, is valid for both independent and positively correlated tests.

To allow for a better comparison with previous studies, the correlations that were strongest using the IAM method were recalculated with spindle measures using a fixed-criterion spindle detection algorithm as common in related research (e.g., Schabus et al., 2007) with fixed threshold frequencies $11-13 \mathrm{~Hz}$ for slow spindles and $13-15 \mathrm{~Hz}$ for fast spindles. Of note, a fixed-criterion method is inherently less sensitive than IAM because it does not take into account individual variations in sleep spindle frequency, instead analyzing a relatively broad frequency band. It is also noteworthy that the fixed-criterion method used somewhat arbitrary detection frequencies, classifying anything $<13 \mathrm{~Hz}$ as a slow spindle and completely missing sleep spindles slower than $11 \mathrm{~Hz}$. This might lead to incorrect classification of spindles in some subjects as well as missed detections of the slowest spindles. However, fast spindle amplitude on a prominent fast-spindle generating site $(\mathrm{Cz})$ is a feature salient enough to be reliable detected even with a less sensitive spindle detection method.

\section{Results}

In general, slow spindle amplitude was highest at frontal derivations (maximum amplitude on $\mathrm{Fz}$ ), whereas fast spindles were most prominent on central and parietal derivations (maximum amplitude on $\mathrm{Cz}$ ). Mean peak frequency was $11.43 \mathrm{~Hz}$ (SD 0.76 $\mathrm{Hz}$, range $9.59-13.28 \mathrm{~Hz}$ ) for slow spindles and $13.72 \mathrm{~Hz}$ (SD $0.59 \mathrm{~Hz}$, range $12.5-15.38 \mathrm{~Hz}$ ) for fast spindles.

Age was in significant negative correlation with all sleep spindle parameters on all electrodes, except for slow spindle amplitude on all electrodes, slow spindle duration on $\mathrm{Cz}, \mathrm{Fz}$, and $\mathrm{T}$, and fast spindle amplitude on $\mathrm{O} 1$ and $\mathrm{O} 2$. Positive correlations between age and slow spindle amplitude were significant on $\mathrm{O} 2$ (median amplitude only) and P4 (both median and maximum amplitude). A significant positive correlation with fast spindle peak frequency and significant negative correlation with Raven equivalent raw scores was also found.

Sex differences were found in various sleep spindle parameters. Women had significantly higher fast spindle amplitudes in derivations F3, F4, Fz, C3, C4, Cz, P3, P4, T6, O1, and O2, and higher peak frequencies both in case of slow and fast spindles. Men had significantly higher fast spindle densities on derivations P3, P4, O1, and $\mathrm{O} 2$, and significantly higher fast spindle durations on $\mathrm{O} 2$.

Sex differences were found in various sleep spindle parameters. Women had significantly higher fast spindle amplitudes in derivations F3 $\left(\mathrm{Mean}_{\text {male }}=4.61, \mathrm{Mean}_{\mathrm{female}}=5.13, t=-2.18\right.$, $p=0.03), \mathrm{F} 4\left(\mathrm{Mean}_{\text {male }}=4.66, \mathrm{Mean}_{\mathrm{female}}=5.3, t=-2.66, p=\right.$ $0.008), \mathrm{Fz}\left(\mathrm{Mean}_{\text {male }}=5.29, \mathrm{Mean}_{\mathrm{female}}=5.99, t=-2.39, p=0.02\right)$, $\mathrm{C} 3\left(\mathrm{Mean}_{\text {male }}=5.20, \mathrm{Mean}_{\mathrm{female}}=5.82, t=-2.55, p=0.01\right), \mathrm{C} 4$ $\left(\mathrm{Mean}_{\text {male }}=5.24, \mathrm{Mean}_{\mathrm{female}}=4.92, t=-2.83, p=0.005\right), \mathrm{Cz}$ $\left(\mathrm{Mean}_{\text {male }}=6.81, \mathrm{Mean}_{\mathrm{female}}=8.02, t=-3.55, p=0.0005\right)$, P3 $\left(\mathrm{Mean}_{\text {male }}=5.43, \mathrm{Mean}_{\text {female }}=6.2, t=-2.99, p=0.003\right), \mathrm{P} 4$ $\left(\mathrm{Mean}_{\text {male }}=5.22, \mathrm{Mean}_{\mathrm{female}}=5.91, t=-2.66, p=0.009\right)$, T6 $\left(\mathrm{Mean}_{\text {male }}=2.97, \mathrm{Mean}_{\mathrm{female}}=3.28, t=-2.07, p=0.04\right)$, $\mathrm{O} 1\left(\mathrm{Mean}_{\text {male }}=3.81, \mathrm{Mean}_{\mathrm{female}}=4.36, t=-2.51, p=0.01\right)$, and $\mathrm{O} 2\left(\mathrm{Mean}_{\text {male }}=3.77, \mathrm{Mean}_{\mathrm{female}}=4.22, t=-2.14, p=0.03\right)$, and higher peak frequencies $(\mathrm{Hz})$ in case of both slow $\left(\mathrm{Mean}_{\text {male }}=\right.$ 11.28, Mean $\left._{\text {female }}=11.61, t=-2.82, p=0.005\right)$ and fast $\left(\right.$ Mean $_{\text {male }}=13.55$, Mean $\left._{\text {female }}=13.92, t=-4.13, p=0.00006\right)$ spindles. Men had significantly higher fast spindle densities (no./ min) on derivations P3 $\left(\right.$ Mean $_{\text {male }}=7.64$, Mean $_{\text {female }}=7.34, t=$ $2.00, p=0.04), \mathrm{P} 4\left(\mathrm{Mean}_{\mathrm{male}}=7.60, \mathrm{Mean}_{\mathrm{female}}=7.30, t=2.00\right.$, $p=0.04), \mathrm{O} 1$ (Mean $_{\text {male }}=7.24$, Mean $_{\mathrm{female}}=6.84, t=2.35, p=$ $0.02)$, and O2 $\left(\right.$ Mean $_{\text {male }}=7.29$, Mean $_{\text {female }}=6.76, t=3.08, p=$ 0.002 ), and significantly higher fast spindle durations on $\mathrm{O} 2$ $\left(\right.$ Mean ${ }_{\text {male }}=1.09$, Mean $\left._{\text {female }}=1.03, t=2.57, p=0.01\right)$.

Strong sex differences were found in correlations between sleep spindle parameters and Raven equivalent scores. In females, 
Table 1. Continued

\begin{tabular}{|c|c|c|c|c|c|}
\hline & Precision & Hardware prefiltering $(\mathrm{Hz})$ & $\begin{array}{l}\text { Amplitude attenuation } \\
10-15 \mathrm{~Hz} \text { (mean [SD]) }\end{array}$ & Recording apparatus & Recording software \\
\hline Budapest-I & 12bit & $0.5-70$ & $0.9705[0.0036]$ & $\begin{array}{l}\text { Flat Style SLEEP La Mont Headbox, HBX32-SLP } \\
\text { preamplifier (La Mont Medical) }\end{array}$ & DataLab (Medcare) \\
\hline Budapest-II & 12 bit & $\begin{array}{l}0.33-1500 \text { ( }<450 \mathrm{~Hz} \text { antialiasing digital } \\
\text { filtering before undersampling) }\end{array}$ & $0.9356[0.0021]$ & Brain-Quick BQ 132S (Micromed) & System 98 (Micromed) \\
\hline Munich-I & 8bit & $0.53-70$ & $0.9693[0.0016]$ & Comlab 32 DigitalSleep Lab & Brainlab, version 3.3 \\
\hline Munich-II & 8 bit & $0.53-70$ & $0.9693[0.0016]$ & Comlab 32 DigitalSleep Lab & Brainlab, version 3.3 \\
\hline
\end{tabular}

Table 2. Partial Pearson correlation coefficients (corrected for age) between sleep spindle parameters and Raven APM scores in female subjects ${ }^{a}$

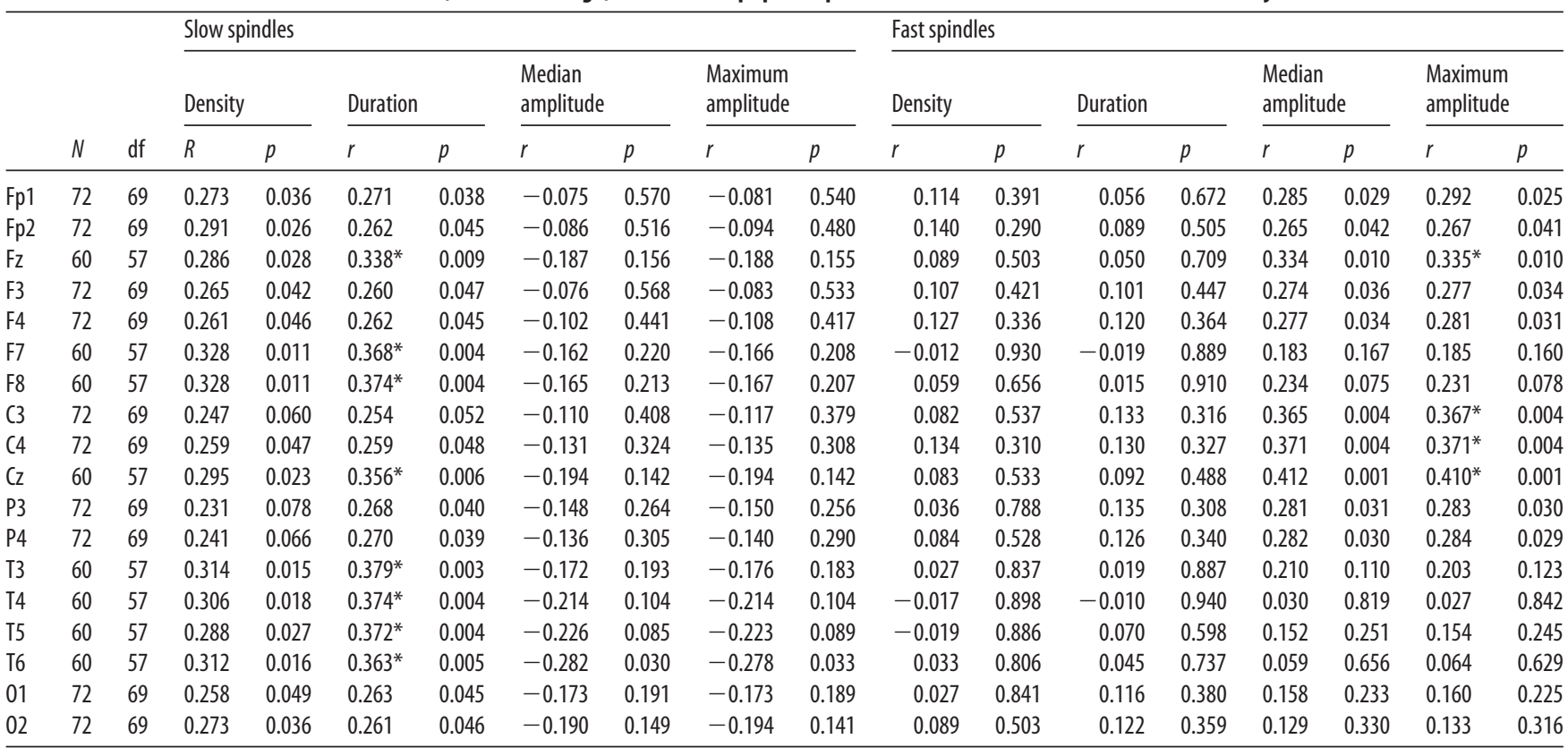

${ }^{a}$ In the first two columns, the number of available subjects and the corresponding degrees of freedom are given for each electrode.

*Correlations that remain significant after multiple comparisons correction.

age-corrected partial correlations were significant between Raven equivalent scores and fast spindle amplitude (central, frontal, and parietal derivations, $\mathrm{r}_{\max }=0.412 \mathrm{on} \mathrm{Cz}$ ) and slow spindle duration (all derivations with the exception of C3, $\mathrm{r}_{\max }=0.379$ on T3). In males, age-corrected partial correlations revealed a negative association between Raven equivalent scores and fast spindle density (posterior derivations, $\mathrm{r}_{\max }=-0.337$ on O1). After correction for multiple testing, partial correlation coefficients were significant between Raven equivalent scores and fast spindle amplitude (electrodes $\mathrm{Cz}, \mathrm{C} 3, \mathrm{C} 4$, and $\mathrm{Fz}$ ) and slow spindle duration (electrodes F7, F8, T3, T4, T5, T6, Cz, and Fz) in females, as well as fast spindle density (electrodes O1, O2, P3, P4, and T5) in males.

Table 2 gives an overview of the partial correlations found in females. Table 3 gives an overview of the partial correlations found in males. Table 4 gives an overview of partial correlations in all subjects. Figure 1 illustrates the most prominent partial correlations between Raven equivalent scores, fast spindle amplitude, slow spindle duration, and fast spindle density in both sexes.

Sex differences in the correlations between Raven equivalent scores and sleep spindle parameters were confirmed by statistical comparison of the maximal significant correlations illustrated in Figure 2. Using Fisher's $r$ to $z$ transformation method, correlation coefficients found in males and females were significantly different for fast spindle amplitude on $\mathrm{Cz}(z=3.2, p=0.001)$, slow spindle duration on T3 $(z=3.23, p=0.001)$, and fast spindle density on $\mathrm{O} 1(z=2.23, p=0.02)$.

Sleep spindle peak frequencies were not correlated with Raven equivalent scores in either sex and in either slow or fast spindles (age-corrected partial correlation with slow spindle peak frequency is 0.17 [ $p=0.160]$ in females, $-0.06[p=0.539]$ in males; correlation with fast spindle peak frequency is $-0.04[p=0.744]$ in females, 0.095 [ $p=0.379]$ in males).

Similar results were seen if individual intelligence test raw scores (CFT or Raven) were used instead of the combined score. Correlations were also not exclusively driven by either subgroup (Budapest or Munich) used in the study (for details, see Fig. 2, scatterplots). Inclusion or exclusion of the 8 smoking subjects did not change the results of the study.

To replicate our main findings with $90 \%$ statistical power, a sample size of $n=48$ would be required for fast spindle amplitude on $\mathrm{Cz}(r=0.41)$, a sample size of $n=56$ would be required for slow spindle duration on T3 $(r=0.371)$, and a sample size of $n=72$ would be required for fast spindle density on $\mathrm{O} 1(r=$ $-0.337)$. Given that these sample sizes are meant for subjects from the same sex due to sex differences in the relationship 
Table 3. Partial Pearson correlation coefficients (corrected for age) between sleep spindle parameters and Raven APM scores in male subjects ${ }^{a}$

\begin{tabular}{|c|c|c|c|c|c|c|c|c|c|c|c|c|c|c|c|c|c|c|}
\hline & \multirow[b]{3}{*}{$N$} & \multirow[b]{3}{*}{$\mathrm{df}$} & \multicolumn{8}{|c|}{ Slow spindles } & \multicolumn{8}{|c|}{ Fast spindles } \\
\hline & & & \multicolumn{2}{|l|}{ Density } & \multicolumn{2}{|l|}{ Duration } & \multicolumn{2}{|c|}{$\begin{array}{l}\text { Median } \\
\text { amplitude }\end{array}$} & \multicolumn{2}{|c|}{$\begin{array}{l}\text { Maximum } \\
\text { amplitude }\end{array}$} & \multicolumn{2}{|l|}{ Density } & \multicolumn{2}{|l|}{ Duration } & \multicolumn{2}{|c|}{$\begin{array}{l}\text { Median } \\
\text { amplitude }\end{array}$} & \multicolumn{2}{|c|}{$\begin{array}{l}\text { Maximum } \\
\text { amplitude }\end{array}$} \\
\hline & & & $r$ & $p$ & $r$ & $p$ & $r$ & $p$ & $r$ & $p$ & $r$ & $p$ & $r$ & $p$ & $r$ & $p$ & $r$ & $p$ \\
\hline Fp1 & 88 & 85 & -0.016 & 0.890 & -0.100 & 0.379 & -0.102 & 0.370 & -0.101 & 0.374 & -0.182 & 0.106 & -0.123 & 0.278 & -0.173 & 0.126 & -0.177 & 0.117 \\
\hline $\mathrm{Fp} 2$ & 88 & 85 & 0.005 & 0.963 & -0.102 & 0.366 & -0.087 & 0.442 & -0.077 & 0.500 & -0.168 & 0.135 & -0.090 & 0.429 & -0.172 & 0.128 & -0.165 & 0.145 \\
\hline $\mathrm{Fz}$ & 81 & 78 & 0.045 & 0.690 & -0.080 & 0.481 & -0.038 & 0.741 & -0.030 & 0.791 & -0.241 & 0.032 & -0.086 & 0.450 & -0.110 & 0.330 & -0.109 & 0.337 \\
\hline F3 & 88 & 85 & 0.043 & 0.707 & -0.083 & 0.466 & -0.065 & 0.565 & -0.057 & 0.617 & -0.241 & 0.031 & -0.107 & 0.345 & -0.124 & 0.274 & -0.125 & 0.271 \\
\hline F4 & 88 & 85 & 0.012 & 0.917 & -0.095 & 0.401 & -0.078 & 0.493 & -0.068 & 0.552 & -0.211 & 0.060 & -0.041 & 0.719 & -0.136 & 0.228 & -0.134 & 0.236 \\
\hline F7 & 81 & 78 & 0.045 & 0.694 & -0.094 & 0.405 & -0.036 & 0.754 & -0.030 & 0.792 & -0.172 & 0.128 & -0.073 & 0.520 & -0.093 & 0.413 & -0.087 & 0.445 \\
\hline F8 & 81 & 78 & 0.027 & 0.815 & -0.108 & 0.341 & -0.078 & 0.492 & -0.071 & 0.530 & -0.167 & 0.139 & -0.027 & 0.816 & -0.145 & 0.199 & -0.134 & 0.237 \\
\hline C 3 & 88 & 85 & 0.096 & 0.395 & -0.099 & 0.384 & -0.073 & 0.518 & -0.065 & 0.568 & -0.238 & 0.033 & -0.127 & 0.262 & -0.114 & 0.316 & -0.110 & 0.331 \\
\hline C4 & 88 & 85 & 0.064 & 0.573 & -0.109 & 0.335 & -0.093 & 0.411 & -0.083 & 0.462 & -0.219 & 0.051 & -0.084 & 0.460 & -0.128 & 0.259 & -0.124 & 0.273 \\
\hline $\mathrm{Cz}$ & 81 & 78 & 0.094 & 0.407 & -0.106 & 0.350 & -0.055 & 0.629 & -0.050 & 0.661 & -0.234 & 0.037 & -0.090 & 0.426 & -0.081 & 0.476 & -0.079 & 0.489 \\
\hline P3 & 88 & 85 & 0.098 & 0.388 & -0.135 & 0.232 & -0.111 & 0.325 & -0.102 & 0.367 & $-0.309^{*}$ & 0.005 & -0.121 & 0.286 & -0.189 & 0.093 & -0.184 & 0.103 \\
\hline P4 & 88 & 85 & 0.122 & 0.282 & -0.118 & 0.296 & -0.059 & 0.602 & -0.051 & 0.656 & $-0.312^{*}$ & 0.005 & -0.128 & 0.260 & -0.138 & 0.222 & -0.134 & 0.236 \\
\hline $\mathrm{T} 3$ & 81 & 78 & 0.085 & 0.456 & -0.124 & 0.272 & -0.117 & 0.301 & -0.117 & 0.302 & -0.165 & 0.144 & -0.066 & 0.563 & -0.190 & 0.092 & -0.182 & 0.107 \\
\hline $\mathrm{T} 4$ & 81 & 78 & 0.062 & 0.584 & -0.121 & 0.285 & -0.134 & 0.235 & -0.125 & 0.271 & -0.162 & 0.150 & -0.055 & 0.626 & -0.197 & 0.080 & -0.163 & 0.148 \\
\hline T5 & 81 & 78 & 0.097 & 0.391 & -0.132 & 0.244 & -0.069 & 0.542 & -0.065 & 0.565 & $-0.287^{*}$ & 0.010 & -0.108 & 0.340 & -0.063 & 0.577 & -0.059 & 0.604 \\
\hline T6 & 81 & 78 & 0.074 & 0.512 & -0.134 & 0.235 & -0.121 & 0.286 & -0.113 & 0.317 & -0.266 & 0.017 & -0.089 & 0.432 & -0.144 & 0.204 & -0.140 & 0.214 \\
\hline 01 & 88 & 85 & 0.083 & 0.462 & -0.140 & 0.216 & -0.081 & 0.477 & -0.071 & 0.531 & $-0.337^{*}$ & 0.002 & -0.140 & 0.216 & -0.128 & 0.256 & -0.125 & 0.268 \\
\hline 02 & 88 & 85 & 0.104 & 0.357 & -0.126 & 0.265 & -0.066 & 0.559 & -0.054 & 0.634 & $-0.315^{*}$ & 0.004 & -0.128 & 0.259 & -0.143 & 0.207 & -0.139 & 0.219 \\
\hline
\end{tabular}

${ }^{a}$ In the first two columns, the number of available subjects and the corresponding degrees of freedom are given for each electrode.

*Correlations that remain significant after multiple comparisons correction.

Table 4. Partial Pearson correlation coefficients (corrected for age) between sleep spindle parameters and Raven APM scores in all subjects ${ }^{a}$

\begin{tabular}{|c|c|c|c|c|c|c|c|c|c|c|c|c|c|c|c|c|c|c|}
\hline & \multirow[b]{3}{*}{$N$} & \multirow[b]{3}{*}{$\mathrm{df}$} & \multicolumn{8}{|c|}{ Slow spindles } & \multicolumn{8}{|c|}{ Fast spindles } \\
\hline & & & \multicolumn{2}{|c|}{ Density } & \multicolumn{2}{|c|}{ Duration } & \multicolumn{2}{|c|}{$\begin{array}{l}\text { Median } \\
\text { amplitude }\end{array}$} & \multicolumn{2}{|c|}{$\begin{array}{l}\text { Maximum } \\
\text { amplitude }\end{array}$} & \multicolumn{2}{|l|}{ Density } & \multicolumn{2}{|l|}{ Duration } & \multicolumn{2}{|c|}{$\begin{array}{l}\text { Median } \\
\text { amplitude }\end{array}$} & \multicolumn{2}{|c|}{$\begin{array}{l}\text { Maximum } \\
\text { amplitude }\end{array}$} \\
\hline & & & $r$ & $p$ & $r$ & $p$ & $r$ & $p$ & $r$ & $p$ & $r$ & $p$ & $r$ & $p$ & $r$ & $p$ & $r$ & $p$ \\
\hline Fp1 & 160 & 157 & 0.127 & 0.135 & 0.073 & 0.393 & -0.083 & 0.327 & -0.085 & 0.321 & -0.039 & 0.650 & -0.025 & 0.769 & 0.038 & 0.659 & 0.035 & 0.685 \\
\hline $\mathrm{Fp} 2$ & 160 & 157 & 0.144 & 0.089 & 0.066 & 0.441 & -0.082 & 0.338 & -0.079 & 0.354 & -0.025 & 0.769 & 0.007 & 0.935 & 0.021 & 0.806 & 0.023 & 0.791 \\
\hline $\mathrm{Fz}$ & 141 & 138 & 0.156 & 0.066 & 0.076 & 0.371 & -0.075 & 0.381 & -0.074 & 0.388 & -0.071 & 0.406 & 0.007 & 0.937 & 0.052 & 0.544 & 0.053 & 0.535 \\
\hline F3 & 160 & 157 & 0.136 & 0.109 & 0.070 & 0.409 & -0.096 & 0.257 & -0.094 & 0.270 & -0.049 & 0.567 & 0.050 & 0.561 & 0.038 & 0.652 & 0.040 & 0.636 \\
\hline $\mathrm{F} 4$ & 160 & 157 & 0.183 & 0.031 & 0.112 & 0.188 & -0.107 & 0.207 & -0.106 & 0.214 & -0.100 & 0.242 & -0.035 & 0.681 & 0.021 & 0.804 & 0.024 & 0.776 \\
\hline F7 & 141 & 138 & 0.168 & 0.047 & 0.106 & 0.211 & -0.129 & 0.129 & -0.126 & 0.137 & -0.077 & 0.365 & -0.001 & 0.995 & 0.011 & 0.902 & 0.015 & 0.861 \\
\hline F8 & 141 & 138 & 0.167 & 0.049 & 0.111 & 0.193 & -0.127 & 0.135 & -0.123 & 0.147 & -0.084 & 0.327 & -0.011 & 0.895 & 0.068 & 0.424 & 0.069 & 0.415 \\
\hline C3 & 160 & 157 & 0.173 & 0.041 & 0.064 & 0.452 & -0.103 & 0.225 & -0.102 & 0.229 & -0.071 & 0.406 & 0.011 & 0.902 & 0.082 & 0.339 & 0.083 & 0.328 \\
\hline (4 & 160 & 157 & 0.160 & 0.059 & 0.060 & 0.481 & -0.121 & 0.156 & -0.118 & 0.165 & -0.042 & 0.626 & 0.034 & 0.690 & 0.076 & 0.372 & 0.078 & 0.359 \\
\hline $\mathrm{Cz}$ & 141 & 138 & 0.198 & 0.019 & 0.103 & 0.224 & -0.143 & 0.093 & -0.140 & 0.099 & -0.078 & 0.360 & 0.005 & 0.954 & 0.094 & 0.269 & 0.095 & 0.264 \\
\hline P3 & 160 & 157 & 0.168 & 0.047 & 0.052 & 0.542 & -0.141 & 0.098 & -0.137 & 0.108 & -0.111 & 0.190 & 0.021 & 0.804 & 0.010 & 0.907 & 0.015 & 0.864 \\
\hline P4 & 160 & 157 & 0.182 & 0.032 & 0.058 & 0.493 & -0.111 & 0.191 & -0.109 & 0.199 & -0.093 & 0.277 & 0.014 & 0.868 & 0.034 & 0.687 & 0.037 & 0.661 \\
\hline $\mathrm{T} 3$ & 141 & 138 & 0.200 & 0.018 & 0.098 & 0.252 & -0.154 & 0.070 & -0.155 & 0.068 & -0.064 & 0.453 & -0.017 & 0.842 & -0.035 & 0.678 & -0.035 & 0.686 \\
\hline $\mathrm{T} 4$ & 141 & 138 & 0.179 & 0.035 & 0.096 & 0.261 & -0.179 & 0.034 & -0.174 & 0.040 & -0.104 & 0.222 & -0.030 & 0.725 & -0.104 & 0.221 & -0.090 & 0.292 \\
\hline T5 & 141 & 138 & 0.198 & 0.019 & 0.089 & 0.295 & -0.171 & 0.044 & -0.167 & 0.049 & -0.127 & 0.134 & -0.006 & 0.943 & -0.003 & 0.974 & -0.001 & 0.996 \\
\hline T6 & 141 & 138 & 0.192 & 0.023 & 0.083 & 0.329 & -0.224 & 0.008 & -0.219 & 0.009 & -0.107 & 0.206 & -0.011 & 0.902 & -0.087 & 0.307 & -0.083 & 0.328 \\
\hline 01 & 160 & 157 & 0.170 & 0.044 & 0.044 & 0.603 & -0.145 & 0.087 & -0.140 & 0.099 & -0.122 & 0.152 & 0.004 & 0.964 & -0.022 & 0.797 & -0.020 & 0.814 \\
\hline 02 & 160 & 157 & 0.184 & 0.030 & 0.051 & 0.551 & -0.147 & 0.083 & -0.142 & 0.095 & -0.062 & 0.468 & 0.017 & 0.839 & -0.036 & 0.671 & -0.033 & 0.697 \\
\hline
\end{tabular}

${ }^{a}$ In the first two columns, the number of available subjects and the corresponding degrees of freedom are given for each electrode. No correlations are significant after multiple comparisons correction.

between IQ and spindles, previous studies appear to have been underpowered.

The correlations that were strongest using the IAM method were recalculated with sleep spindle parameters calculated by a fixed amplitude detection method. In this method, we filtered data for the individual IAM frequencies and applied a fixed amplitude threshold set at $4 \mu \mathrm{V}$ for slow spindles and $6 \mu \mathrm{V}$ for fast spindles. These amplitude criteria were defined based on the 12 $\mu \mathrm{V}$ amplitude limit of the Somnolyzer SIESTA fixed amplitude spindle detection method (Anderer et al., 2005) but reduced because of the narrower frequency bands used in IAM. The amplitude criteria were kept intentionally high to reduce false-positive detections in subjects with high baseline EEG voltage: therefore, depending on the electrode, 1-8 female and 2-12 male subjects provided zero detections and were not considered for the analysis of spindle parameters on the given electrode.

Data from this alternative approach successfully replicated our main finding: the age-corrected partial correlation between intelligence and fast spindle median amplitude on $\mathrm{Cz}$ was significant after correction for multiple testing in females $(N=71, r=$ $0.41, p=0.001)$, but not in males $(N=79, r=0.005, p=0.965)$. Furthermore, we found a significant positive correlation between intelligence and fast spindle density on $\mathrm{C} 4, \mathrm{Cz}$, and $\mathrm{Fz}$ in females $\left(N=71\right.$ for $\mathrm{C} 4, N=59$ for $\mathrm{Cz}$ and $\mathrm{Fz}, \mathrm{r}_{\max }=0.373$ on $\mathrm{Cz}, p=$ 


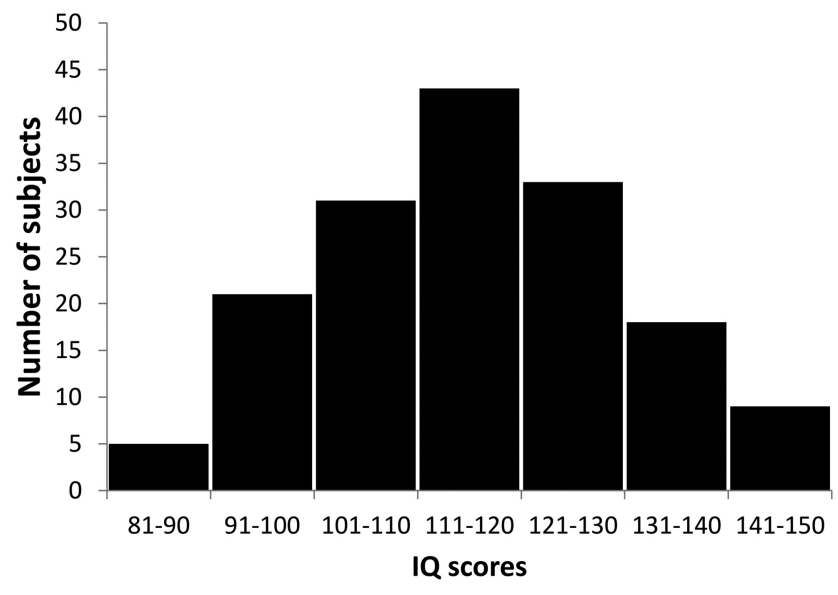

Figure 1. Distribution of $\mathrm{IQ}$ scores in the sample.

$0.004)$ as well as a positive correlation between intelligence and fast spindle duration on $\mathrm{O} 1$ and $\mathrm{O} 2$ in males $\left(N=81, \mathrm{r}_{\max }=\right.$ 0.356 on $\mathrm{O} 2, p=0.002$ ). Other spindle parameters did not correlate significantly with intelligence scores after multiple testing correction in either sex.

\section{Discussion}

Different sleep spindle parameters have repeatedly been associated with state and trait measures of cognitive variables (Fogel and Smith, 2011). However, typical study samples consisted of a rather small number of university students, leading to results that rely on a restricted variance in intelligence scores. Testing the stability of the correlates of intelligence over the entire intelligence range requires the inclusion of a relatively high number of subjects representing also the extremes of the IQ distribution. In our study, we analyzed data of 160 subjects, including many subjects with high and very high intelligence scores. Our findings did not indicate decreasingly linear associations within the extreme IQ ranges, suggesting that the functional and physiological significance of sleep spindles is consistent across the entire intelligence distribution. In contrast to the proposed U-shaped association between intelligence and sleep spindle activity (Fogel et al., 2007; Fogel and Smith, 2011), we found this association to be linear.

In line with previous research (Principe and Smith, 1982; Dijk et al., 1989; Landolt et al., 1996; Carrier et al., 2001; Nicolas et al., 2001; Bódizs et al., 2009), our study revealed a generally negative correlation between age and most measures of sleep spindle activity, and a positive correlation between age and spindle frequency. Slow spindles appeared to be less affected by aging, as for these we found no negative correlation with amplitude (indeed, there was a positive correlation on $\mathrm{O} 2$ and $\mathrm{P} 4$, which however did not survive correction for multiple comparisons) and on some electrodes $(\mathrm{Cz}, \mathrm{Fz}, \mathrm{T} 5)$ no negative correlation with duration either. This suggests that, whereas slow spindle density and (in most cases) duration decrease with age, amplitude is preserved, supporting previous evidence about different neural mechanisms being involved in slow and fast spindles (Schabus et al., 2007). The absence of correlations with fast spindle amplitudes at occipital derivations probably stems from an already low level of spindle activity on these nonprominent spindle locations in a younger age.

Previous research has never specifically looked for sex differences in the sleep EEG correlates of intelligence. However, striking sex differences are known not only for brain anatomy in general (Cahill, 2006; Gong et al., 2009; Jazin and Cahill, 2010;
Tomasi and Volkow, 2012; Ingalhalikar et al., 2014; Satterthwaite et al., 2014) and sleep spindle activity (Huupponen et al., 2002), but specifically for neural correlates of intelligence as measured by waking EEG (Neubauer et al., 2002; Jausovec and Jausovec, 2005) or brain anatomy (Gur et al., 1999; Haier et al., 2005). In addition, sleep spindle activity differentially affects memory consolidation in males and females (Genzel et al., 2012). Most notably, white matter microstructure was shown to be closely associated with sleep spindle activity (Piantoni et al., 2013) and intelligence in females, but not in males (Gur et al., 1999; Haier et al., 2005). In line with these observations, our analyses revealed marked sex differences in the association between intelligence and sleep spindle activity: we found a correlation between Raven equivalent scores and fast sleep spindle amplitudes in central derivations in females, but not in males. Our findings suggest that thalamocortical connections underlying EEG sleep spindle activity are associated with intelligence in females, but not in males.

The positive association between Raven equivalent scores and slow spindle durations was generally present at almost all scalp locations, most prominent in temporal derivations, and found exclusively in females. Sleep spindles coalesce with cortical slow oscillations (Steriade, 2003), and the strength of this temporal synchrony between slow oscillation up-states and sleep spindles was shown to be positively correlated with intellectual performance (Bódizs et al., 2005). A positive relationship between slowwave upstate length and memory consolidation has also been demonstrated (Heib et al., 2013). Based on these findings, our results suggest that longer slow sleep spindle durations in more intelligent females might reflect more precise coalescence between sleep spindles and cortical slow oscillations or easier elicitability of spindles by such oscillations.

The correlation between intelligence and slow spindle duration was maximal in temporal derivations. Regional sleep spindles at a certain cortical location are not necessarily byproducts of sleep spindles at more prominent locations but arise from the activity of local corticothalamic oscillations (Nir et al., 2011), and localized sleep spindles play a role in local synaptic plasticity and subsequent sleep-related learning (Nishida and Walker, 2007). Generally, strong neural activation increases related to slow spindles have been demonstrated for the temporal lobe (Schabus et al., 2007). The lateral frontal and temporal maximum of our findings likely has functional importance, suggesting that, in females, longer sleep spindle durations are connected to intelligence mainly in cortical areas responsible for higher-order visual processing and language. Interestingly, structural imaging studies have found a positive correlation between intelligence in females and white matter volume in Broca's area (Haier et al., 2005), an overlapping and at least in part functionally similar (language-related) brain region.

In males, a negative association between Raven equivalent scores and fast spindle density was found, limited to posterior scalp locations with a left occipital maximum. This finding conflicts with earlier research with samples, including both males and females (Fogel and Smith, 2011), however, is in line with a study of male rats that found learning potential to be negatively associated with spindle density (Fogel et al., 2010). Based on the topography and locally limited nature of our findings, it might be speculated that lower occipital fast spindle density in highly intelligent males is due to higher neural efficiency of the lower-level visual areas. Of note, also in human waking EEG, evidence for higher neural efficiency being associated with intelligence was found in males only (Jausovec and Jausovec, 2005; Neubauer and Fink, 2009). 

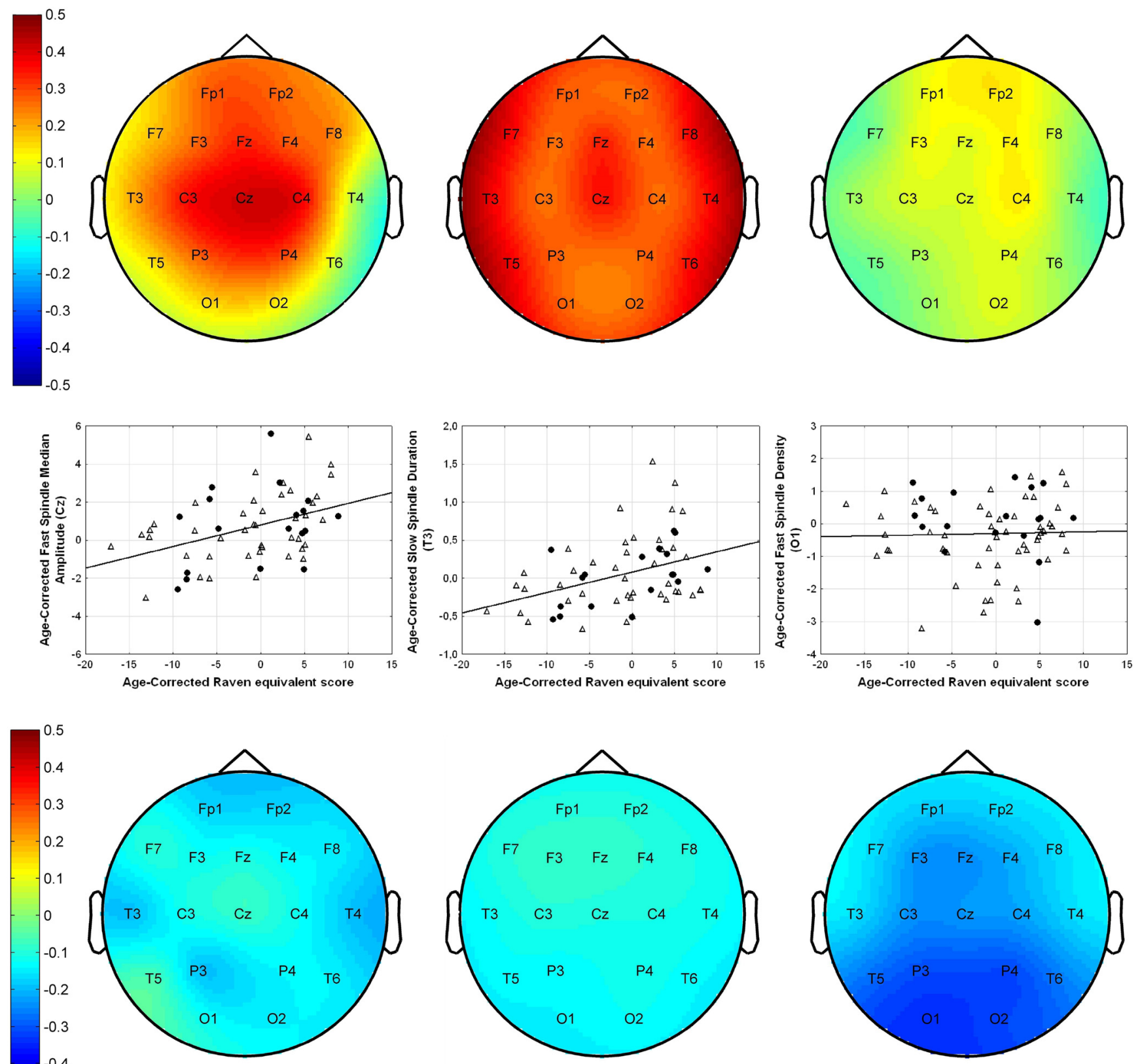

$-0.5$
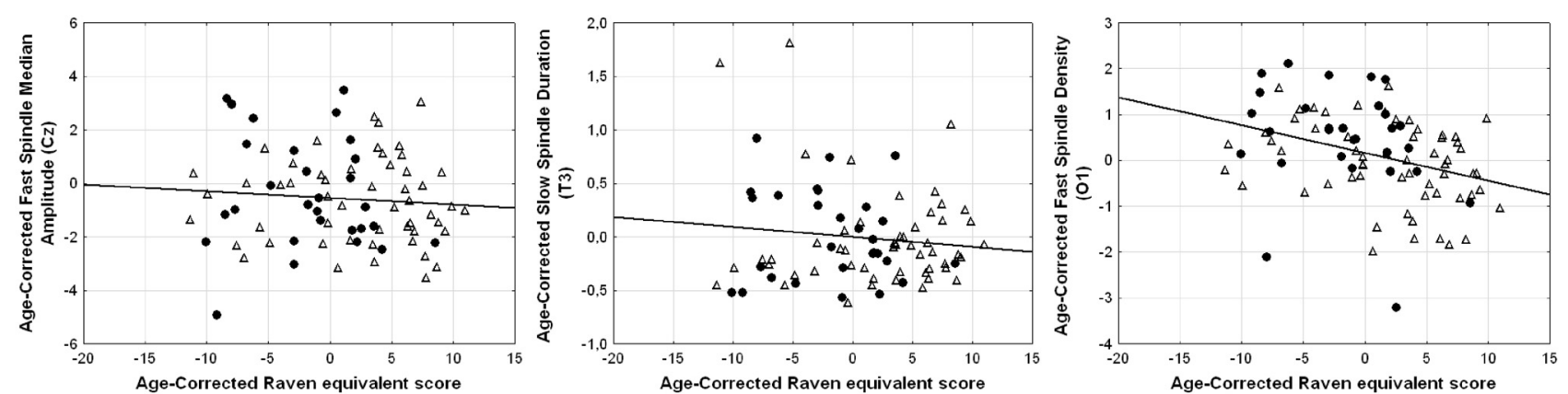

Figure 2. Scalp maps and partial regression plots for females (top half) and males (bottom half), for Cz fast spindle median amplitude (left), T3 slow spindle duration (middle), and 01 fast spindle density (right). Scalp maps illustrate the topographical distribution of the strength of partial correlations between Raven equivalent scores and sleep spindle parameters. On the partial regression plots, $x$-axes represent the residuals after regressing Raven APM scores against age. $y$-axes represent the residuals after regressing spindle parameters against age. Thus, these scatterplots demonstrate the relationship between Raven APM scores and spindle parameters after pruning both variables for the effects of age. Standard Pearson correlation between the shown residuals equals the age-corrected partial correlations between Raven APM scores and spindle parameters. Dots represent data points from the Budapest sample. Triangles represent data points from the Munich sample. 
Table 5. Earlier studies reporting associations between sleep spindle parameters and intelligence ${ }^{a}$

\begin{tabular}{|c|c|c|c|c|c|c|}
\hline Reference & Test & Age & Sex & Spindle parameter & Electrode & Correlation \\
\hline Bodizs et al., 2005 & RPMT:IQ & $27-67$ years & $5 f / 14 m$ & Density slow & $\begin{array}{c}\mathrm{Fp} 1, \mathrm{Fp} 2, \mathrm{Fpz}, \mathrm{F3}, \mathrm{F4}, \mathrm{Fz}, \mathrm{F} 7, \mathrm{~F} 8, \mathrm{C} 3, \mathrm{C} 4, \mathrm{Cz}, \\
\mathrm{T} 3, \mathrm{~T} 4, \mathrm{~T} 5, \mathrm{~T} 6, \mathrm{P3}, \mathrm{P} 4, \mathrm{Pz}, 01,02,0 \mathrm{z}\end{array}$ & NS \\
\hline Bodizs et al., 2005 & RPMT:IQ & $27-67$ years & $5 f / 14 m$ & Density fast & $\begin{array}{c}\mathrm{Fp} 1, \mathrm{Fp} 2, \mathrm{Fpz}, \mathrm{F3}, \mathrm{F4}, \mathrm{Fz}, \mathrm{F} 7, \mathrm{F8}, \mathrm{C3}, \mathrm{C} 4, \mathrm{Cz}, \\
\mathrm{T} 3, \mathrm{~T} 4, \mathrm{~T} 5, \mathrm{~T} 6, \mathrm{P3}, \mathrm{P4}, \mathrm{Pz}, 01,02,0 \mathrm{z}\end{array}$ & $\begin{array}{c}\text { From } r=0.25, p=0.33 \\
\text { at } 02 \text { to } r=0.79, \\
p=0.0001 \text { at Fp2 }\end{array}$ \\
\hline Clemens et al., 2006 & RPMT:IQ & $25-47$ years, mean 35, SD 7.7 & $15 \mathrm{~m}$ & Total number & $\begin{array}{c}\mathrm{Fp} 1, \mathrm{Fpz}, \mathrm{Fp} 2, \mathrm{~F} 7, \mathrm{F3}, \mathrm{Fz}, \mathrm{F} 4, \mathrm{~F} 8, \mathrm{~T} 3, \mathrm{C} 3, \mathrm{Cz} \\
\mathrm{C4}, \mathrm{T4}, \mathrm{T} 5, \mathrm{P3}, \mathrm{Pz}, \mathrm{P4}, \mathrm{T} 6,01,0 \mathrm{Oz}, 02\end{array}$ & NS \\
\hline Schabus et al., 2006 & APM: IQ & $20-30$ years, mean 24, SD 2.6 & $24 \mathrm{f} / 24 \mathrm{~m}$ & Activity slow & C3 & $r=0.40, p<0.01$ \\
\hline Schabus et al., 2006 & APM: IQ & $20-30$ years, mean 24, SD 2.6 & $24 \mathrm{f} / 24 \mathrm{~m}$ & Activity fast & C3 & $r=0.44, p<0.01$ \\
\hline Schabus et al., 2006 & APM: IQ & $20-30$ years, mean 24, SD 2.6 & $24 \mathrm{f} / 24 \mathrm{~m}$ & Density slow & $\mathrm{C} 3$ & $r=0.06, p=0.68$ \\
\hline Schabus et al., 2006 & APM: IQ & $20-30$ years, mean 24, SD 2.6 & $24 \mathrm{f} / 24 \mathrm{~m}$ & Density fast & $\mathrm{C} 3$ & $r=-0.01, p=0.97$ \\
\hline Schabus et al., 2006 & APM: IQ & $20-30$ years, mean 24, SD 2.6 & $24 \mathrm{f} / 24 \mathrm{~m}$ & Duration slow & C3 & $r=0.09, p=0.54$ \\
\hline Schabus et al., 2006 & APM: IQ & $20-30$ years, mean 24, SD 2.6 & $24 \mathrm{f} / 24 \mathrm{~m}$ & Duration fast & $\mathrm{C} 3$ & $r=0.34, p=0.02$ \\
\hline Schabus et al., 2006 & APM: IQ & $20-30$ years, mean 24, SD 2.6 & $24 \mathrm{f} / 24 \mathrm{~m}$ & Amplitude slow & $\mathrm{C} 3$ & $r=0.39, p=0.01$ \\
\hline Schabus et al., 2006 & APM: IQ & $20-30$ years, mean 24, SD 2.6 & $24 \mathrm{f} / 24 \mathrm{~m}$ & Amplitude fast & C3 & $r=0.35, p=0.02$ \\
\hline Fogel et al., 2007 & MAB-II: VIQ & $18-29$ years & $10 \mathrm{f}$ & Total number & $\mathrm{C} 3, \mathrm{C} 4$ & $r=0.56, p=0.09$ \\
\hline Fogel et al., 2007 & MAB-II: PIQ & $18-29$ years & $10 f$ & Total number & $\mathrm{C} 3, \mathrm{C} 4$ & $r=71, p=0.02$ \\
\hline Fogel et al., 2007 & MAB-II: FSIQ & $18-29$ years & $10 f$ & Total number & $\mathrm{C} 3, \mathrm{C} 4$ & $r=0.76, p=0.01$ \\
\hline Fogel et al., 2007 & MAB-II: VIQ & $20-25$ years & $12 f$ & Total number & $\mathrm{C} 3, \mathrm{C} 4$ & $r=0.38, p=0.10$ \\
\hline Fogel et al., 2007 & MAB-II: PIQ & $20-25$ years & $12 f$ & Total number & $\mathrm{C} 3, \mathrm{C}$ & $r=0.79, p=0.001$ \\
\hline Fogel et al., 2007 & MAB-II: VIQ & $20-25$ years & $12 f$ & Total number & $\mathrm{Cz}$ & $r=0.01, p=0.94$ \\
\hline Fogel et al., 2007 & MAB-II: PIQ & $20-25$ years & $12 f$ & Total number & $\mathrm{Cz}$ & $r=0.05, p=0.79$ \\
\hline Fogel et al., 2007 & MAB-II: VIQ & $18-26$ years, mean 20, SD 5.3 & $29 \mathrm{f} / 6 \mathrm{~m}$ & Density & $\mathrm{Cz}$ & NS \\
\hline Fogel et al., 2007 & MAB-II: PIQ & $18-26$ years, mean 20, SD 5.3 & $29 \mathrm{f} / 6 \mathrm{~m}$ & Density & $\mathrm{Cz}$ & NS \\
\hline Fogel et al., 2007 & MAB-II: VIQ & $18-26$ years, mean 20, SD 5.3 & $29 \mathrm{f} / 6 \mathrm{~m}$ & Duration & $\mathrm{Cz}$ & NS \\
\hline Fogel et al., 2007 & MAB-II: PIQ & $18-26$ years, mean 20, SD 5.3 & $29 \mathrm{f} / 6 \mathrm{~m}$ & Duration & $\mathrm{Cz}$ & NS \\
\hline Peters et al., 2007 & MAB-II:VIQ & Mean 21, SD 2.4 & $12 \mathrm{f} / 12 \mathrm{~m}$ & Density & $\mathrm{C} 3, \mathrm{C} 4$ & $r=-0.26, p>0.05$ \\
\hline Peters et al., 2007 & MAB-II: PIQ & Mean 21, SD 2.4 & $12 \mathrm{f} / 12 \mathrm{~m}$ & Density & $\mathrm{C} 3, \mathrm{C} 4$ & $r=0.05, p>0.05$ \\
\hline Peters et al., 2007 & MAB-II: FSIQ & Mean 21, SD 2.4 & $12 \mathrm{f} / 12 \mathrm{~m}$ & Density & $\mathrm{C} 3, \mathrm{C} 4$ & $r=-0.11, p>0.05$ \\
\hline Peters et al., 2008 & MAB-II: VIQ & 17-24 years, mean 20, SD 2.3 & $7 f / 7 m$ & Density & $\mathrm{C} 3, \mathrm{C} 4$ & NS \\
\hline Peters et al., 2008 & MAB-II: PIQ & $17-24$ years, mean 20, SD 2.3 & $7 f / 7 m$ & Density & $\mathrm{C} 3, \mathrm{C} 4$ & NS \\
\hline Peters et al., 2008 & MAB-II: FSIQ & 17-24 years, mean 20, SD 2.3 & $7 \mathrm{f} / 7 \mathrm{~m}$ & Density & $\mathrm{C} 3, \mathrm{C} 4$ & NS \\
\hline Peters et al., 2008 & MAB-II: VIQ & 62-79 years, mean 70, SD 5.1 & $7 f / 7 m$ & Density & $\mathrm{C} 3, \mathrm{C} 4$ & NS \\
\hline Peters et al., 2008 & MAB-II: PIQ & 62-79 years, mean 70, SD 5.1 & $7 \mathrm{f} / 7 \mathrm{~m}$ & Density & $\mathrm{C} 3, \mathrm{C} 4$ & NS \\
\hline Peters et al., 2008 & MAB-II: FSIQ & 62-79 years, mean 70, SD 5.1 & $7 f / 7 m$ & Density & $\mathrm{C} 3, \mathrm{C} 4$ & NS \\
\hline Tucker and Fishbein, 2009 & MAB-II: VIQ & Mean 21 years & $12 f / 12 m$ & Sigma power & $\mathrm{C} 3, \mathrm{C} 4$ & NS \\
\hline Tucker and Fishbein, 2009 & MAB-II: PIQ & Mean 21 years & $12 f / 12 m$ & Sigma power & $\mathrm{C} 3, \mathrm{C} 4$ & NS \\
\hline Tucker and Fishbein, 2009 & MAB-II: FSIQ & Mean 21 years & $12 f / 12 m$ & Sigma power & $\mathrm{C} 3, \mathrm{C} 4$ & NS \\
\hline Geiger et al., 2011 & WISC-IV: VIQ & $9-13$ years, mean 10.5 & $6 \mathrm{f} / 8 \mathrm{~m}$ & Spindle peak frequency & $\mathrm{C} 3, \mathrm{C}$ & NS \\
\hline Geiger et al., 2011 & WISC-IV: FIQ & $9-13$ years, mean 10.5 & $6 f / 8 m$ & Spindle peak frequency & $\mathrm{C} 3, \mathrm{C} 4$ & NS \\
\hline Geiger et al., 2011 & WISC-IV: FSIQ & $9-13$ years, mean 10.5 & $6 \mathrm{f} / 8 \mathrm{~m}$ & Spindle peak frequency & $\mathrm{C} 3, \mathrm{C} 4$ & $r=-0.56, p<0.05$ \\
\hline Geiger et al., 2011 & WISC-IV: VIQ & $9-13$ years, mean 10.5 & $6 \mathrm{f} / 8 \mathrm{~m}$ & Sigma power & $\mathrm{C} 3, \mathrm{C} 4$ & NS \\
\hline Geiger et al., 2011 & WISC-IV: FIQ & $9-13$ years, mean 10.5 & $6 \mathrm{f} / 8 \mathrm{~m}$ & Sigma power & $\mathrm{C} 3, \mathrm{C} 4$ & $r=0.65, p<0.05$ \\
\hline Geiger et al., 2011 & WISC-IV: FSIQ & $9-13$ years, mean 10.5 & $6 \mathrm{f} / 8 \mathrm{~m}$ & Sigma power & $\mathrm{C} 3, \mathrm{C} 4$ & $r=0.67, p<0.01$ \\
\hline Lustenberger et al., 2012 & ZVT: IQ & $18-20$ years, mean 19, SD 0.8 & $15 \mathrm{~m}$ & Activity & C4 & $r=0.55, p<0.05$ \\
\hline Chatburn et al., 2013 & SBIS: VIQ & 4-13 years, mean 8, SD 2.1 & $13 f / 14 m$ & Total number: all, fast, slow & $\mathrm{C}, \mathrm{C} 4$ & NS \\
\hline Chatburn et al., 2013 & SBIS: NVIQ & $4-13$ years, mean 8, SD 2.1 & $13 \mathrm{f} / 14 \mathrm{~m}$ & Total number: all, fast, slow & $\mathrm{C} 3, \mathrm{C} 4$ & NS \\
\hline Chatburn et al., 2013 & SBIS: FSIQ & $4-13$ years, mean 8, SD 2.1 & $13 \mathrm{f} / 14 \mathrm{~m}$ & Total number: all, fast, slow & $\mathrm{C} 3, \mathrm{C} 4$ & NS \\
\hline Chatburn et al., 2013 & SBIS: VIQ & $4-13$ years, mean $8, S D 2.1$ & $13 \mathrm{f} / 14 \mathrm{~m}$ & Density: all, fast, slow & $\mathrm{C} 3, \mathrm{C} 4$ & NS \\
\hline Chatburn et al., 2013 & SBIS: NVIQ & $4-13$ years, mean 8, SD 2.1 & $13 \mathrm{f} / 14 \mathrm{~m}$ & Density: all, fast, slow & $\mathrm{C}, \mathrm{C} 4$ & NS \\
\hline Chatburn et al., 2013 & SBIS: FSIQ & $4-13$ years, mean $8, S D 2.1$ & $13 f / 14 m$ & Density: all, fast, slow & $\mathrm{C} 3, \mathrm{C} 4$ & NS \\
\hline Chatburn et al., 2013 & SBIS: VIQ & $4-13$ years, mean 8, SD 2.1 & $13 \mathrm{f} / 14 \mathrm{~m}$ & Duration & $\mathrm{C} 3, \mathrm{C} 4$ & NS \\
\hline Chatburn et al., 2013 & SBIS: NVIQ & $4-13$ years, mean 8, SD 2.1 & $13 \mathrm{f} / 14 \mathrm{~m}$ & Duration & $\mathrm{C} 3, \mathrm{C} 4$ & NS \\
\hline Chatburn et al., 2013 & SBIS: FSIQ & $4-13$ years, mean 8, SD 2.1 & $13 \mathrm{f} / 14 \mathrm{~m}$ & Duration & $\mathrm{C} 3, \mathrm{C} 4$ & NS \\
\hline Chatburn et al., 2013 & SBIS: VIQ & $4-13$ years, mean $8, S D 2.1$ & $13 \mathrm{f} / 14 \mathrm{~m}$ & Frequency & $\mathrm{C} 3, \mathrm{C} 4$ & NS \\
\hline Chatburn et al., 2013 & SBIS: NVIQ & 4-13 years, mean 8, SD 2.1 & $13 f / 14 m$ & Frequency & $\mathrm{C} 3, \mathrm{C} 4$ & NS \\
\hline Chatburn et al., 2013 & SBIS: FSIQ & $4-13$ years, mean $8, S D 2.1$ & $13 \mathrm{f} / 14 \mathrm{~m}$ & Frequency & $\mathrm{C}, \mathrm{C} 4$ & NS \\
\hline Gruber et al., 2013 & WISC-IV: FSIQ & $7-11$ years, mean 9, SD 0.9 & $14 \mathrm{f} / 15 \mathrm{~m}$ & Density & $\mathrm{F} 3, \mathrm{~F} 4, \mathrm{C} 3, \mathrm{C} 4, \mathrm{P} 3, \mathrm{P} 4,01,02$ & NS \\
\hline Gruber et al., 2013 & WISC-IV: FSIQ & 7-11 years, mean 9, SD 0.9 & $14 \mathrm{f} / 15 \mathrm{~m}$ & Amplitude & $\mathrm{F} 3, \mathrm{~F} 4, \mathrm{C} 3, \mathrm{C} 4, \mathrm{P3}, \mathrm{P4}, 01,02$ & NS \\
\hline Gruber et al., 2013 & WISC-IV: FSIQ & 7-11 years, mean 9, SD 0.9 & $14 \mathrm{f} / 15 \mathrm{~m}$ & Duration & $\mathrm{F} 3, \mathrm{~F} 4, \mathrm{C} 3, \mathrm{C} 4, \mathrm{P} 3, \mathrm{P} 4,01,02$ & NS \\
\hline Gruber et al., 2013 & WISC-IV: FSIQ & 7-11 years, mean 9, SD 0.9 & $14 \mathrm{f} / 15 \mathrm{~m}$ & Frequency & $\mathrm{F} 3, \mathrm{~F} 4, \mathrm{C} 3, \mathrm{C} 4, \mathrm{P} 3, \mathrm{P} 4,01,02$ & NS \\
\hline Ward et al., 2014 & MAB-II: VIQ & $18-29$ years, mean 21, SD 3.0 & $21 \mathrm{f} / 9 \mathrm{~m}$ & Density & C3 & $r=0.18, p>0.05$ \\
\hline Ward et al., 2014 & MAB-II: PIQ & $18-29$ years, mean 21, SD 3.0 & $21 \mathrm{f} / 9 \mathrm{~m}$ & Density & C3 & $r=0.14, p>0.05$ \\
\hline Ward et al., 2014 & MAB-II: FSIQ & $18-29$ years, mean 21, SD 3.0 & $21 \mathrm{f} / 9 \mathrm{~m}$ & Density & C3 & $r=0.22, p>0.05$ \\
\hline
\end{tabular}


Earlier studies on the relationship between sleep spindle parameters and cognition (e.g., Schabus et al., 2006, 2008) have used fixed-amplitude methods for sleep spindle detection (Anderer et al., 2005). These methods do not correct for intraindividual differences in baseline EEG voltage, which on the one hand renders them more sensitive to absolute differences in sleep spindle amplitude, but on the other hand potentially introduces noise due to the influence of non-neural effects (such as skull thickness) on EEG signal amplitude. Despite these differences, our results were successfully, though with weaker effects, replicated using a fixed-amplitude spindle detection algorithm: Both fast spindle amplitude and fast spindle density (technically speaking, the proportion of fast spindles over the critical amplitude) are significantly correlated with IQ in females, but not males.

Although our study is limited in its ability to reveal the precise reason for such sex differences, previous research suggests genetically determined differences in brain anatomy and endocrine function to play a role. On the one hand, sleep EEG features are known to be genetically determined (De Gennaro et al., 2008; Landolt, 2011), and this genetic determination manifests itself largely through variations in brain anatomy and structure (Smit et al., 2012), in which there are notable variations between males and females (Cahill, 2006; Gong et al., 2009; Jazin and Cahill, 2010; Tomasi and Volkow, 2012; Ingalhalikar et al., 2014; Satterthwaite et al., 2014). On the other hand, estrogen and progesterone levels in females, as well as the 2-4 digit ratio (a sensitive correlate of female sex hormones) were shown to be directly associated with sleep spindle features and sleep-related cognitive measures (Driver et al., 1996; Genzel et al., 2012).

Overall, our results only partially confirm previous literature about the positive relationship between intelligence and sleep spindle parameters: We found such positive associations with intelligence for sleep spindle amplitude and sleep spindle length only, and exclusively in females. The focus of many earlier reports of the relationship between sleep spindles and cognitive processes was originally on state markers of sleep-related memory consolidation and not on trait markers, such as intelligence or learning capacity. Such publications of findings originally unintended in the study design have recently been suggested to be prone to publication bias, such as the file drawer effect: results consistent with mainstream views are more probable to be submitted (and accepted), whereas negative findings are mentioned less prominently (Cordi et al., 2014). Indeed, a closer look into the literature reveals a very mixed picture. An early study found fast, but not slow, spindle density to be associated with intelligence (Bódizs et al., 2005). In contrast, three more recent studies did not find significant correlations between fast spindle density and intelligence (Peters et al., 2007, 2008; Ward et al., 2014; K. Peters, personal communication). Of note, however, the former study observed the strongest correlations in frontal and frontopolar regions, whereas the latter analyzed central derivations only. Studies mainly with female participants reported a significant correlation between the total number of sleep spindles and performance IQ but not verbal IQ; however, in one of the samples, this association held only for the upper IQ range (Fogel et al., 2007), and a further study found neither full scale, nor performance, nor verbal IQ to be correlated with sleep spindle $\sigma$ band (Tucker and Fishbein, 2009). In contrast to these studies, a study with a considerable sample size found spindle duration $\times$ amplitude, but neither total spindle number nor spindle density to be correlated with intelligence (Schabus et al., 2006). Of note, a reanalysis of this study suggests that similar sex differences, as presented here, with a much stronger age-corrected positive cor- relation between IQ and fast spindle amplitude in females ( $M$. Schabus, personal communication). Although also mental speed as an intelligence-related capacity has been found to correlate with spindle duration $\times$ amplitude (Lustenberger et al., 2012), two studies did not find any correlation between sleep spindle activity and working memory (Limoges et al., 2013; Lafortune et al., 2014), despite being closely associated with fluid intelligence (Conway et al., 2003). Sleep spindle activity has been suggested as a marker of normal intellectual development (Shibagaki et al., 1982); however, in healthy children, a relationship between intelligence and sleep spindles has only been reported for spindle peak frequency (Geiger et al., 2011; Gruber et al., 2013), $\sigma$ power (Geiger et al., 2012), or not at all (Chatburn et al., 2013).

In sum, only a minority of studies found significant correlations between intelligence and spindle activity (for an overview, see Table 5). Studies with positive results differed markedly in the specific spindle parameters that were correlated with intelligence: number, density, amplitude, length, power, or peak frequency are considered of undifferentiated, slow, or fast sleep spindles (with considerable variability in definitions); some studies further differentiate between the whole night, night halves, thirds, or quarters, and many studies differentially analyzed several EEG derivations. Also, different spindle detection algorithms have been used, with the less sensitive fixed-amplitude spindle detection being most common. In our study, a control analysis with this method has yielded only partially similar results to the IAM spindle detection method; it failed to replicate IAM findings related to slow spindles and spindles on less prominent spindlegenerating locations. All in all, the large number of potential spindle variables found in the literature may have potentially led to methodological problems due to multiple comparisons. The above-mentioned publication bias applies in particular to studies with smaller sample sizes and hence low statistical power. All of these problems may have led to false positive or false negative findings in earlier studies.

Most notably, none of the cited studies has targeted potential sex differences, which might have obscured potential correlations. Our results suggest that synaptic plasticity through sleep spindle activity is only one, but not the only possible sleep-related neural mechanism underlying intelligence, and it is mostly present in females, possibly because of their more prominent and functionally more important structural brain connectivity. The general relationship between sleep spindles and intelligence may have been overestimated in the previous literature. Future studies should apply a theoretically grounded and welldefined array of sleep spindle parameters and account for potential sex differences.

\section{References}

Amzica F, Steriade M (2000) Integration of low-frequency sleep oscillations in corticothalamic networks. Acta Neurobiol Exp 60:229-245. Medline

Anderer P, Gruber G, Parapatics S, Woertz M, Miazhynskaia T, Klösch G, Saletu B, Zeitlhofer J, Barbanoj MJ, Danker-Hopfe H, Himanen SL, Kemp B, Penzel T, Grözinger M, Kunz D, Rappelsberger P, Schlögl A, Dorffner G (2005) An E-Health solution for automatic sleep classification according to Rechtschaffen and Kales: validation dtudy of the Somnolyzer $24 \times 7$ utilizing the Siesta Database. Neuropsychobiology 51:115-133. CrossRef Medline

Benjamini Y, Hochberg Y (1995) Controlling the false discovery rate: a practical and powerful approach to multiple testing. J R Stat Soc B 289300 .

Bixler EO, Rhodes JM (1968) Spindle activity during sleep in culturalfamilial mild retardates. Psychophysiology 5:212.

Bódizs R, Kis T, Lazar AS, Havrán L, Rigó P, Clemens Z, Halász P (2005) 
Prediction of general mental ability based on neural oscillation measures of sleep. J Sleep Res 14:285-292. CrossRef Medline

Bódizs R, Lazár A, Rigó P (2008) Correlation of visuospatial memory ability with right parietal EEG spindling during sleep. Acta Physiol Hung 95: 297-306. CrossRef Medline

Bódizs R, Körmendi J, Rigó P, Lázár AS (2009) The individual adjustment method of sleep spindle analysis: methodological improvements and roots in the fingerprint paradigm. J Neurosci Methods 178:205-213. CrossRef Medline

Buzsáki G (1989) Two-stage model of memory trace formation: a role for "noisy" brain states. Neuroscience 31:551-570. CrossRef Medline

Cahill L (2006) Why sex matters for neuroscience. Nat Rev Neurosci 7:477484. CrossRef Medline

Carrier J, Land S, Buysse DJ, Kupfer DJ, Monk TH (2001) The effects of age and gender on sleep EEG power spectral density in the middle years of life (ages 20-60 years old). Psychophysiology 38:232-242. CrossRef Medline

Cattell RB (1973) Culture fair intelligence test: (a measure of "g"). Champaign, IL: Institute for Personality and Ability Testing.

Chatburn A, Coussens S, Lushington K, Kennedy D, Baumert M, Kohler M (2013) Sleep spindle activity and cognitive performance in healthy children. Sleep 36:237-243. CrossRef Medline

Clemens Z, Fabó D, Halász P (2005) Overnight verbal memory retention correlates with the number of sleep spindles. Neuroscience 132:529-535. CrossRef Medline

Clemens Z, Fabó D, Halász P (2006) Twenty-four hours retention of visuospatial memory correlates with the number of parietal sleep spindles. Neurosci Lett 403:52-56. CrossRef Medline

Clemens Z, Mölle M, Eross L, Barsi P, Halász P, Born J (2007) Temporal coupling of parahippocampal ripples, sleep spindles and slow oscillations in humans. Brain 130:2868-2878. CrossRef Medline

Conway AR, Kane MJ, Engle RW (2003) Working memory capacity and its relation to general intelligence. Trends Cogn Sci 7:547-552. CrossRef Medline

Cordi M, Ackermann S, Bes FW, Hartmann F, Konrad BN, Genzel L, Pawlowski M, Steiger A, Schulz H, Rasch B, Dresler M (2014) Lunar cycle effects on sleep and the file drawer problem. Curr Biol 24:R549-R550. CrossRef Medline

De Gennaro L, Ferrara M (2003) Sleep spindles: an overview. Sleep Med Rev 7:423-440. CrossRef Medline

De Gennaro L, Ferrara M, Vecchio F, Curcio G, Bertini M (2005) An electroencephalographic fingerprint of human sleep. Neuroimage 26:114122. CrossRef Medline

De Gennaro L, Marzano C, Fratello F, Moroni F, Pellicciari MC, Ferlazzo F, Costa S, Couyoumdjian A, Curcio G, Sforza E, Malafosse A, Finelli LA, Pasqualetti P, Ferrara M, Bertini M, Rossini PM (2008) The electroencephalographic fingerprint of sleep is genetically determined: a twin study. Ann Neurol 64:455-460. CrossRef Medline

Dijk DJ, Beersma DG, van den Hoofdakker RH (1989) All night spectral analysis of EEG sleep in young adult and middle-aged male subjects. Neurobiol Aging 10:677-682. CrossRef Medline

Driver HS, Dijk DJ, Werth E, Biedermann K, Borbély AA (1996) Sleep and the sleep electroencephalogram across the menstrual cycle in young healthy women. J Clin Endocrinol Metab 81:728-735. CrossRef Medline

Duncan J, Seitz RJ, Kolodny J, Bor D, Herzog H, Ahmed A, Newell FN, Emslie H (2000) A neural basis for general intelligence. Science 289:457-460. CrossRef Medline

Fogel SM, Smith CT (2006) Learning-dependent changes in sleep spindles and Stage 2 sleep. J Sleep Res 15:250-255. CrossRef Medline

Fogel SM, Smith CT (2011) The function of the sleep spindle: a physiological index of intelligence and a mechanism for sleep-dependent memory consolidation. Neurosci Biobehav Rev 35:1154-1165. CrossRef Medline

Fogel SM, Nader R, Cote KA, Smith CT (2007) Sleep spindles and learning potential. Behav Neurosci 121:1-10. CrossRef Medline

Fogel SM, Smith CT, Beninger RJ (2010) Too much of a good thing? Elevated baseline sleep spindles predict poor avoidance performance in rats. Brain Res 1319:112-117. CrossRef Medline

Forest G, Poulin J, Daoust AM, Lussier I, Stip E, Godbout R (2007) Attention and non-REM sleep in neuroleptic-naive persons with schizophrenia and control participants. Psychiatry Res 149:33-40. CrossRef Medline

Gaillard JM, Blois R (1981) Spindle density in sleep of normal subjects. Sleep 4:385-391. Medline
Gais S, Mölle M, Helms K, Born J (2002) Learning-dependent increases in sleep spindle density. J Neurosci 22:6830-6834. Medline

Geiger A, Huber R, Kurth S, Ringli M, Jenni OG, Achermann P (2011) The sleep EEG as a marker of intellectual ability in school age children. Sleep 34:181-189. Medline

Geiger A, Huber R, Kurth S, Ringli M, Achermann P, Jenni OG (2012) Sleep electroencephalography topography and children's intellectual ability. Neuroreport 23:93-97. CrossRef Medline

Genzel L, Dresler M, Wehrle R, Grözinger M, Steiger A (2009) Slow wave sleep and REM sleep awakenings do not affect sleep dependent memory consolidation. Sleep 32:302-310. Medline

Genzel L, Kiefer T, Renner L, Wehrle R, Kluge M, Grözinger M, Steiger A, Dresler M (2012) Sex and modulatory menstrual cycle effects on sleep related memory consolidation. Psychoneuroendocrinology 37:987-998. CrossRef Medline

Genzel L, Kroes MC, Dresler M, Battaglia FP (2014) Light sleep versus slow wave sleep in memory consolidation: a question of global versus local processes? Trends Neurosci 37:10-19. CrossRef Medline

Gibbs EL, Gibbs FA (1962) Extreme spindles: correlation of electroencephalographic sleep pattern with mental retardation. Science 138:1106-1107. CrossRef Medline

Gong G, He Y, Concha L, Lebel C, Gross DW, Evans AC, Beaulieu C (2009) Mapping anatomical connectivity patterns of human cerebral cortex using in vivo diffusion tensor imaging tractography. Cereb Cortex 19:524536. CrossRef Medline

Gruber R, Wise MS, Frenette S, Knäauper B, Boom A, Fontil L, Carrier J (2013) The association between sleep spindles and IQ in healthy schoolage children. Int J Psychophysiol 89:229-240. CrossRef Medline

Gur RC, Turetsky BI, Matsui M, Yan M, Bilker W, Hughett P, Gur RE (1999) Sex differences in brain gray and white matter in healthy young adults: correlations with cognitive performance. J Neurosci 19:4065-4072. Medline

Haier RJ, Jung RE, Yeo RA, Head K, Alkire MT (2005) The neuroanatomy of general intelligence: sex matters. Neuroimage 25:320-327. CrossRef Medline

Heib DP, Hoedlmoser K, Anderer P, Zeitlhofer J, Gruber G, Klimesch W, Schabus M (2013) Slow oscillation amplitudes and up-state lengths relate to memory improvement. PLoS One 8:e82049. CrossRef Medline

Huupponen E, Himanen SL, Värri A, Hasan J, Lehtokangas M, Saarinen J (2002) A study on gender and age differences in sleep spindles. Neuropsychobiology 45:99-105. CrossRef Medline

Iber C (2007) The American Academy of Sleep Medicine Manual for the scoring of sleep and associated events: rules, terminology and technical specification. Darien, IL: American Academy of Sleep Medicine.

Ingalhalikar M, Smith A, Parker D, Satterthwaite TD, Elliott MA, Ruparel K, Hakonarson H, Gur RE, Gur RC, Verma R (2014) Sex differences in the structural connectome of the human brain. Proc Natl Acad Sci U S A 111:823-828. CrossRef Medline

Jaspers K (1958) The physician in the technical age. Klin Wochenschr 36: 1037-1043. CrossRef Medline

Jausovec N, Jausovec K (2005) Sex differences in brain activity related to general and emotional intelligence. Brain Cogn 59:277-286. CrossRef Medline

Jazin E, Cahill L (2010) Sex differences in molecular neuroscience: from fruit flies to humans. Nat Rev Neurosci 11:9-17. CrossRef Medline

Lafortune M, Gagnon JF, Martin N, Latreille V, Dubé J, Bouchard M, Bastien C, Carrier J (2014) Sleep spindles and rapid eye movement sleep as predictors of next morning cognitive performance in healthy middle-aged and older participants. J Sleep Res 23:159-167. CrossRef Medline

Landolt HP (2011) Genetic determination of sleep EEG profiles in healthy humans. Prog Brain Res 193:51-61. CrossRef Medline

Landolt HP, Dijk DJ, Achermann P, Borbély AA (1996) Effect of age on the sleep EEG: slow-wave activity and spindle frequency activity in young and middle-aged men. Brain Res 738:205-212. CrossRef Medline

Limoges É, Bolduc C, Berthiaume C, Mottron L, Godbout R (2013) Relationship between poor sleep and daytime cognitive performance in young adults with autism. Res Dev Disabil 34:1322-1335. CrossRef Medline

Lustenberger C, Maric A, Durr R, Achermann P, Huber R (2012) Triangular relationship between sleep spindle activity, general cognitive ability and the efficiency of declarative learning. PLoS One 7:21. CrossRef Medline

Morin A, Doyon J, Dostie V, Barakat M, Hadj Tahar A, Korman M, Benali H, Karni A, Ungerleider LG, Carrier J (2008) Motor sequence learning in- 
creases sleep spindles and fast frequencies in post-training sleep. Sleep 31:1149-1156. Medline

Neubauer AC, Fink A (2009) Intelligence and neural efficiency: measures of brain activation versus measures of functional connectivity in the brain. Intelligence 37:223-229. CrossRef

Neubauer AC, Fink A, Schrausser DG (2002) Intelligence and neural efficiency: the influence of task content and sex on the brain-IQ relationship. Intelligence 30:515-536. CrossRef

Nicolas A, Petit D, Rompré S, Montplaisir J (2001) Sleep spindle characteristics in healthy subjects of different age groups. Clin Neurophysiol 112: 521-527. CrossRef Medline

Nir Y, Staba RJ, Andrillon T, Vyazovskiy VV, Cirelli C, Fried I, Tononi G (2011) Regional slow waves and spindles in human sleep. Neuron 70: 153-169. CrossRef Medline

Nishida M, Walker MP (2007) Daytime naps, motor memory consolidation and regionally specific sleep spindles. PLoS One 2:e341. CrossRef Medline

Peters KR, Smith V, Smith CT (2007) Changes in sleep architecture following motor learning depend on initial skill level. J Cogn Neurosci 19:817829. CrossRef Medline

Peters KR, Ray L, Smith V, Smith C (2008) Changes in the density of stage 2 sleep spindles following motor learning in young and older adults. J Sleep Res 17:23-33. CrossRef Medline

Peyrache A, Khamassi M, Benchenane K, Wiener SI, Battaglia FP (2009) Replay of rule-learning related neural patterns in the prefrontal cortex during sleep. Nat Neurosci 12:919-926. CrossRef Medline

Piantoni G, Poil SS, Linkenkaer-Hansen K, Verweij IM, Ramautar JR, Van Someren EJ, Van Der Werf YD (2013) Individual differences in white matter diffusion affect sleep oscillations. J Neurosci 33:227-233. CrossRef Medline

Principe JC, Smith JR (1982) Sleep spindle characteristics as a function of age. Sleep 5:73-84. Medline

Prokosch MD, Yeo RA, Miller GF (2005) Intelligence tests with higher g-loadings show higher correlations with body symmetry: evidence for a general fitness factor mediated by developmental stability. Intelligence 33:203-213. CrossRef

Raven JC, Raven J, Court JH (2004) Raven Haladó Progresszív Mátrixok Kézikönyv. Budapest, Hungary: Edge2000 Kft.

Rosanova M, Ulrich D (2005) Pattern-specific associative long-term potentiation induced by a sleep spindle-related spike train. J Neurosci 25:93989405. CrossRef Medline

Satterthwaite TD, Elliott MA, Ruparel K, Loughead J, Prabhakaran K, Calkins ME, Hopson R, Jackson C, Keefe J, Riley M, Mentch FD, Sleiman P, Verma R, Davatzikos C, Hakonarson H, Gur RC, Gur RE (2014) Neu- roimaging of the Philadelphia Neurodevelopmental Cohort. Neuroimage 86:544-553. CrossRef Medline

Schabus M, Hödlmoser K, Gruber G, Sauter C, Anderer P, Klösch G, Parapatics S, Saletu B, Klimesch W, Zeitlhofer J (2006) Sleep spindle-related activity in the human EEG and its relation to general cognitive and learning abilities. Eur J Neurosci 23:1738-1746. CrossRef Medline

Schabus M, Dang-Vu TT, Albouy G, Balteau E, Boly M, Carrier J, Darsaud A, Degueldre C, Desseilles M, Gais S, Phillips C, Rauchs G, Schnakers C, Sterpenich V, Vandewalle G, Luxen A, Maquet P (2007) Hemodynamic cerebral correlates of sleep spindles during human non-rapid eye movement sleep. Proc Natl Acad Sci U S A 104:13164-13169. CrossRef Medline

Shibagaki M, Kiyono S, Watanabe K, Hakamada S (1982) Concurrent occurrence of rapid eye movement with spindle burst during nocturnal sleep in mentally retarded children. Electroencephalogr Clin Neurophysiol 53:27-35. CrossRef Medline

Smit DJ, Boomsma DI, Schnack HG, Hulshoff Pol HE, de Geus EJ (2012) Individual differences in EEG spectral power reflect genetic variance in gray and white matter volumes. Twin Res Hum Genet 15:384-392. CrossRef Medline

Spearman C (1927) The abilities of man. Oxford, United Kingdom: Macmillan.

Steriade M (2000) Corticothalamic resonance, states of vigilance and mentation. Neuroscience 101:243-276. CrossRef Medline

Steriade M (2003) The corticothalamic system in sleep. Front Biosci $1: \mathrm{d} 878-\mathrm{d} 899$

Steriade M, Deschenes M (1984) The thalamus as a neuronal oscillator. Brain Res 320:1-63. Medline

Tomasi D, Volkow ND (2012) Gender differences in brain functional connectivity density. Hum Brain Mapp 33:849-860. CrossRef Medline

Tucker MA, Fishbein W (2009) The impact of sleep duration and subject intelligence on declarative and motor memory performance: how much is enough? J Sleep Res 18:304-312. CrossRef Medline

Tucker-Drob EM (2009) Differentiation of cognitive abilities across the life span. Dev Psychol 45:1097-1118. CrossRef Medline

Ward MP, Peters KR, Smith CT (2014) Effect of emotional and neurtral declarative memory consolidation on sleep architecture. Exp Brain Res 232:1525-1534. CrossRef Medline

Weiss R, Weiss B (2006) CFT-20R Grundintelligenzstest Skala, Revision 2. Göttingen, The Netherlands: Hogrefe Verlag.

Wierzynski CM, Lubenov EV, Gu M, Siapas AG (2009) State-dependent spike-timing relationships between hippocampal and prefrontal circuits during sleep. Neuron 61:587-596. CrossRef Medline 